\title{
Computational performance of a single bandpass electro-optic delay oscillator
}

\author{
L. Appeltant ${ }^{1}$, G. Van der Sande ${ }^{1}$, S. Rybalko ${ }^{2}$, R. Martinenghi ${ }^{2}$, M. Jacquot ${ }^{2}$, Y. Chembo ${ }^{2}$, L. Larger ${ }^{2}$, \\ I. Fischer ${ }^{3}$, J. Danckaert ${ }^{1}$ \\ 1. Applied Physics Research Group (APHY), Vrije Universiteit Brussel, Pleinlaan 2, B-1050 Brussel, Belgium \\ 2. UMR CNRS 6174, FEMTO-ST / Optics, University of Franche-Comté, 16 route de Gray, 25030, Besancon cedex, France \\ 3. Instituto de Física Interdisciplinar y Sistemas Complejos, IFISC (UIB-CSIC), Campus Universitat de les Illes Balears, E-07122 Palma de \\ Mallorca, Spain
}

Reservoir computing has recently been put forward as the generic name of a new research line in machine learning [1]. This field combines Echo State Networks, introduced by Jaeger [2], and Liquid State Machines, introduced by Maass [3]. These systems, that can be used to solve complex classification problems in a computationally efficient way, were proposed as an alternative to the recurrent neural networks that depend on very intensive and time-consuming training algorithms. Reservoir computing systems have two distinct parts: the reservoir, and an output layer. Traditionally, the reservoir consists of a vast number of randomly interconnected nodes, receiving input signals. The output layer is a linear weighted sum of the node states with the weights optimised by learning. In practice, networks of several hundred nonlinear nodes often have a good performance.

Within the framework of the EC project PHOCUS a new approach to implement the reservoir has been identified: a single nonlinear node with delayed feedback substitutes an entire network of nodes maintaining similar processing power. By defining virtual nodes as delayed states that reside in the delay line, it suffices to have only one physically present hardware node in the setup. This drastically facilitates the experimental implementations of network structures for classification or prediction tasks.

Here, we perform numerical simulations to determine the performance of a Ikeda-type nonlinearity with delayed feedback. The Ikeda nonlinearity is often encountered in electro-optical systems. One system that can be considered consists of an electrically tunable DBR multielectrode laser diode with a feedback loop formed by a delay line and an optical device, whose peculiarity is to exhibit a nonlinearity in wavelength. This system was proposed by Goedgebuer and coworkers [4].

Specifically the system can be described by an integro-differential equation of the form:

$$
\dot{x}(t)=-x(t)-\frac{1}{T} \int x(s) d s+\eta \cdot \sin ^{2}[x(t-\tau)+\gamma J(t)+\phi],
$$

where $\eta$ is the feedback gain, $\gamma$ is the input scaling, $J$ is the input signal and $\phi$ defines the operating point of the nonlinearity. The system is characterized by a high cutoff frequency with a response time normalized to 1 and a low cutoff frequency with a response time $T$. The optoelectronic feedback also consists of a significant delay $\tau$. It was already identified that the interplay of these three time scales gives rise to a large variety of oscillatory regimes and complex dynamical behaviors [5]. We will discuss how this multiple time scale dynamics and more specifically the bandwidth of the bandpass filtering creates an adequate virtual interconnection structure allowing for classification and computation. The performance of this system is quantified using the NARMA10 task, a time series prediction task targetting only one step, that is considered to be a standard benchmark in the reservoir computing field. We obtain performances comparable to those of the state of the art traditional neural networks, which employ many nonlinear nodes.

\section{References}

[1] Verstraeten, D., Schrauwen, B., DHaene, M., Stroobandt, D., "An experimental unification of reservoir computing methods", Neural Networks 20(3), 391-403 (2007).

[2] Jaeger, H., Haas, H., "Harnessing nonlinearity: predicting chaotic systems and saving energy in wireless communication." Science 304, 78-80 (2004).

[3] Maass, W., Natschläger, T., and Markram, H., "Real-time computing without stable states: A new framework for neural computation based on perturbations". Neural Comp. 14(11), 2531-2560 (2002).

[4] Goedgebuer, J.P., Larger, L., and Porte, H., "Optical Cryptosystem Based on Synchronization of Hyperchaos Generated by a Delayed Feedback Tunable Laser Diode" Phys. Rev. Lett. 80, 2249-2252 (1998)

[5] Peil, M., Jacquot, M., Chembo, Y.K., Larger, L., and Erneux, T., "Routes to chaos and multiple time scale dynamics in broadband bandpass nonlinear delay electro-optic oscillators", Phys. Rev. E 79026208 (2009) 